A review of documents assessing capacity and treatment needs to safeguard adults with incapacity

Tereza Hoggard ${ }^{1 *}$, Robin Holliday ${ }^{2}$ and Everett Julyan ${ }^{2}$

${ }^{1}$ University of Glasgow and ${ }^{2}$ NHS Ayrshire and Arran

${ }^{*}$ Corresponding author.

doi: 10.1192/bjo.2021.255

Aims. To audit the completion of Adults with Incapacity (AWI) documents (Assessment of Capacity, Section 47 Certificate of Incapacity and Treatment Plan) to ensure they met the legal standards required. We hypothesised that the forms were not all completed comprehensively, particularly with regards to the Treatment Plans.

Method. In addition to being legal documents, AWI documents provide an important framework to guide clinicians when giving treatment and balancing patient safety with patient autonomy. Correctly completed documents help provide vulnerable patients with ethical and lawful treatment that allows them to be treated with respect and dignity.

An audit was conducted across two Old Age Psychiatry wards at Ayrshire Central Hospital during October 2020. We assessed all AWI documents available on the wards $(n=20)$ using criteria based on the standards set by the Mental Welfare Commission for Scotland to ensure legal competence.

Result. 95\% of the forms were signed and dated, and the nature of the incapacity was given in $100 \%$ of the documents. On the other hand, 35\% of the forms gave no indication of the presence or absence of a guardian. Only one of those identified as having a guardian was consulted with regards to the treatment plan. Another member of staff was consulted on the Treatment Plan in $45 \%$ of cases. $30 \%$ of the Treatment Plans were not precisely worded enough to be considered justifiable for treatment. In the Certificate of Incapacity, two out-of-date certificates were found, and staff were notified immediately. $45 \%$ of certificates were considered over-generalised with regards to the description under medical treatment.

Conclusion. Overall, the forms were mostly signed and dated, with the nature of incapacity given. The two areas that appeared to be the most problematic were the issue of identifying and discussing plans with a guardian, and the specification of treatment covered by both the Certificate of Incapacity and the Treatment Plan.

Discussion with members of the healthcare team found some confusion over how to complete the forms and many cited a lack of formal training as the main reason for their uncertainty. In addition, accessing clear information online or on the wards on how to complete the forms was challenging. We intend to improve the completion of these documents by implementing teaching and a guidance poster, based on the areas that we identified as being problematic, and completing the audit cycle.

\section{An audit of ECG monitoring in patients admitted to the general adult wards at clock view hospital}

Declan Hyland $^{1 \star}$, Unmol Thandi ${ }^{2}$, Udani Mahamithawa ${ }^{2}$, Yasmine Elagamy ${ }^{3}$ and Mohammed Uddin ${ }^{4}$

${ }^{1}$ Consultant Psychiatrist, Clock View Hospital, Liverpool, Mersey Care NHS Foundation Trust; ${ }^{2} 5$ th year medical undergraduate, University of Liverpool; ${ }^{3}$ Core Trainee 1 in Psychiatry, Clock View Hospital, Liverpool, Mersey Care NHS Foundation Trust and ${ }^{4}$ Physician Associate, Clock View Hospital, Liverpool, Mersey Care NHS Foundation Trust ${ }^{*}$ Corresponding author.

doi: 10.1192/bjo.2021.256
Aims. To identify whether patients admitted to the general adult inpatient wards at Clock View Hospital, an inpatient unit in Mersey Care NHS Foundation Trust, have an ECG performed following admission and whether, if this done, the ECG report is properly documented in the patient's electronic record, and whether those patients with an abnormal ECG have any further investigations requested.

Background. An important risk factor for development of cardiac disease is use of psychotropic medications. Antipsychotics can increase the QTc interval.

NICE guidelines recommend that, before starting antipsychotic medication, an ECG should be offered if physical examination identifies cardiovascular risk factors, there is personal history of cardiac disease or if the individual is being admitted to hospital. The Royal College of Physicians states all patients should be assessed for cardiovascular disease, including having a routine ECG. Mersey Care's physical health policy recommends all new admissions to inpatient wards have an ECG performed within 24 hours of admission as part of their admission physical examination and investigation.

Method. A sample of 60 patients discharged from the general adult wards at Clock View Hospital between 16th of July 2019 and 30th of September 2019 was obtained. An audit tool was designed and each patient's electronic record scrutinised to determine whether an ECG was performed within 24 hours of admission; in those patients who didn't, whether the reason why was recorded; and whether those patients who had an abnormal ECG were referred for further investigation. The quality of documentation of ECG reports was also analysed.

Result. Age range of patients was 19-66 years. Only 31 patients had an ECG performed within 24 hours of admission. Of the remaining 29, there was documentation of why an ECG was not performed in only 16 cases. Thirteen patients had an abnormal ECG, but only three were referred for further investigation. Of the ECG reports that were analysed, only a minority met the required standard for "good", with there being no documentation of the ECG report in one third of cases.

Conclusion. There is significant room for improvement in performance of ECG monitoring and documentation of the ECG report. The importance of the ECG as part of the admission process needs to be highlighted in the induction of junior doctors. Training nursing staff on the wards to perform ECGs would reduce the likelihood of unnecessary delay to a patient having an ECG done following admission.

An audit of high-dose and combination antipsychotic prescribing across the general adult inpatient wards in Mersey Care NHS Foundation Trust

Declan Hyland $^{1 \star}$, Beth Hemmings ${ }^{2}$ and Yasmine Elagamy ${ }^{3}$

${ }^{1}$ Consultant Psychiatrist, Clock View Hospital, Liverpool, Mersey Care NHS Foundation Trust; ${ }^{2} 5$ th year medical undergraduate, University of Liverpool and ${ }^{3}$ Core Trainee 1 in Psychiatry, Clock View Hospital, Liverpool, Mersey Care NHS Foundation Trust ${ }^{*}$ Corresponding author.

doi: 10.1192/bjo.2021.257

Aims. To review the number of prescriptions of regular high-dose antipsychotics and combination antipsychotic therapy across the eight general adult inpatient wards in Mersey Care NHS Foundation Trust and examine whether these prescriptions followed Trust recommendations for high-dose antipsychotic therapy (HDAT).

Background. The two main rationales behind prescribing HDAT are pharmacokinetics differ in individuals and so insufficient 
amounts of antipsychotic may reach the effect site at maximum dose in some patients and variations in the effect site between patients may mean higher doses are required to achieve therapeutic effect.

Method. The electronic prescription records for all patients on the eight general adult inpatient wards were scrutinised. 121 patients were prescribed antipsychotic medication. Any patients on a combination of regular antipsychotic medication or on HDAT were identified. Any patient on combination therapy or HDAT was studied to determine if Clozapine had been considered. The electronic notes of HDAT patients were analysed to ascertain whether tests recommended by Trust guidelines - BMI, blood pressure (B.P), pulse rate, ECG, FBC, U and Es, LFTs, serum prolactin, serum cholesterol and $\mathrm{HbA} 1 \mathrm{c}$ level had been performed prior to initiation and following any dose increase.

Result. 21 of 121 patients prescribed antipsychotic medication were on combination therapy. 11 were subject to HDAT. 8 of the 11 HDAT patients were on combination therapy. Clozapine was considered before initiating HDAT in 9 of the HDAT patients. Clozapine was considered in 13 of the 21 patients on combination antipsychotic therapy, but only two were initiated on Clozapine (combined with Olanzapine or Risperidone).

$100 \%$ of HDAT patients had an ECG prior to initiation of HDAT; only $36 \%$ had one after dose increases above BNF maximum. $100 \%$ of HDAT patients had their BMI measured before initiation. 91\% had baseline B.P and heart rate checked. Of the recommended blood tests, $100 \%$ of HDAT patients had baseline FBC, U and Es, LFTs and serum cholesterol. Fewer patients had a baseline HbA1c level (91\%) or serum prolactin (46\%) measured. Conclusion. Prevalence of HDAT across the general adult inpatient wards in the Trust was $9 \%$, much lower than the $28 \%$ reported in the HDAT audit completed by the Prescribing Observatory for Mental Health in 2012. Patients within Mersey Care are more likely to be prescribed combination therapy than HDAT. Not every HDAT patient has been considered for Clozapine. There is a need to ensure Trust monitoring guidelines for HDAT patients are being strictly adhered to.

An audit to assess the measurement of Body Mass Index (BMI) and referral to the dietetics service following admission to the general adult inpatient wards in Mersey Care NHS Foundation Trust

Declan Hyland ${ }^{1 *}$, Millie Prime ${ }^{2}$ and Rabia Khaliq ${ }^{2}$

${ }^{1}$ Consultant Psychiatrist, Clock View Hospital, Liverpool, Mersey Care NHS Foundation Trust and ${ }^{2} 5$ th year medical undergraduate, University of Liverpool

${ }^{*}$ Corresponding author.

doi: 10.1192/bjo.2021.258

Aims. This audit aims to establish whether patients have their BMI measured and recorded following admission to the general adult inpatient wards in Mersey Care NHS Foundation Trust and whether, in those with a BMI $>30 \mathrm{~kg} / \mathrm{m} 2$, or $>28 \mathrm{~kg} / \mathrm{m} 2$ in those with weight-related comorbidities, they are referred to the dietetics service for input.

Background. Obesity has an increased prevalence in those with mental disorder. There are many factors that influence this, e.g. sedentary lifestyle and poor dietary intake. Medication prescribed to treat mental disorders may increase risk of weight gain. Patients with severe mental illness are at increased likelihood of developing weight-related comorbidities, particularly type II diabetes mellitus.

Many patients with severe and enduring mental illness do not regularly access primary care services. Admission to the psychiatric ward therefore provides an opportunity to address, not only the patient's mental health issues, but also any physical health issues. Method. A list of all inpatients on the eight general adult wards was obtained on 3rd of December 2020. Inpatients on the Psychiatric Intensive Care Unit were also incorporated, providing a final sample of 148 inpatients.

An audit tool was designed, to collect demographic data for each inpatient - gender, age, ethnicity, psychiatric diagnosis, as well as BMI on admission and, if applicable, whether a referral to the dietetics service was made.

Result. Of the 148 inpatients, 91 were male, 57 female. Patient age ranged from 19 to 71 years. The majority were of "white British" ethnicity. The most common mental disorder diagnosis was schizophrenia (35 patients). For 14 of the 148 inpatients, no BMI was measured on admission. In the 134 inpatients that had BMI measured, 74 were in one of the "overweight", "obese", "very obese" and "morbidly obese" categories. Thirty-four patients met the criteria for requiring referral to the dietetics service. Of these, four were not referred, five were offered referral but declined, 17 referrals were made for other reasons, e.g. BMI $<18 \mathrm{~kg} / \mathrm{m} 2$, and one patient was referred despite no BMI being recorded.

Conclusion. Current practice across the general adult inpatient wards in the trust indicates a proportion of patients do not have BMI recorded following admission. This may result in a valuable opportunity to address obesity being lost. There is a need to emphasise to ward staff the importance of recording BMI as part of the admission physical health screen and of the criteria for referring an inpatient to the dietetics service.

\section{An evaluation of the prevalence of weight-related} comorbidities in patients following admission to the general adult inpatient wards in Mersey Care NHS Foundation Trust

Declan Hyland ${ }^{1 \star}$, Millie Prime ${ }^{2}$ and Rabia Khaliq ${ }^{2}$

${ }^{1}$ Consultant Psychiatrist, Clock View Hospital, Liverpool, Mersey Care NHS Foundation Trust and ${ }^{2} 5$ th year medical undergraduate, University of Liverpool

${ }^{\star}$ Corresponding author.

doi: 10.1192/bjo.2021.259

Aims. This audit aims to evaluate the prevalence of any weight-related comorbidities in patients following their admission to the general adult inpatient wards in Mersey Care NHS Foundation Trust.

Background. Obesity has an increased prevalence in those with mental disorder. There are many factors that influence this, e.g. sedentary lifestyle and poor dietary intake. Medication prescribed to treat mental disorders may increase risk of weight gain, particularly most of the second generation antipsychotics. Patients with severe mental illness are at increased likelihood of developing weight-related comorbidities - essential hypertension, ischaemic heart disease, hyperlipidaemia and type II diabetes mellitus.

Many patients with severe and enduring mental illness do not readily or regularly access primary care services. Admission to the psychiatric ward therefore provides an ideal opportunity to address, not only the patient's mental health issues, but also any physical health issues.

Method. A list of all inpatients on the eight general adult wards was obtained on 3rd of December 2020. Inpatients on the Psychiatric Intensive Care Unit were also incorporated, providing a final sample of 148 inpatients.

An audit tool was designed, to collect demographic data for each inpatient - gender, age, ethnicity, psychiatric diagnosis, as 